\title{
Pedestal and ELM characteristics with different first wall materials and nitrogen seeding in ASDEX Upgrade
}

P. A. Schneider, L. Barrera Orte, A. Burckhart, M. G. Dunne, C. Fuchs, A. Gude, B. Kurzan, W. Suttrop, E. Wolfrum and and the ASDEX Upgrade Team

Max-Planck-Institut für Plasmaphysik, Boltzmannstr. 2, 85748 Garching, Germany

\begin{abstract}
A comparison of ASDEX Upgrade (AUG) discharges performed with carbon and the full tungsten wall shows that the pedestal performance at low triangularity is not altered without gas puffing. The pedestal electron pressure is the same for both wall materials as is the confinement. With the tungsten wall the natural density is higher even without an additional gas puff. In typical operation with gas puffing the density is again higher in tungsten. This results in a higher collisionality with the tungsten wall. Pedestal pressure and plasma confinement, however, are not degraded until very large amounts of deuterium are puffed.

The ELM crash in typical AUG discharges is observed to be composed of two independent phases. This is observed for both the carbon and the tungsten wall. The $1^{\text {st }}$ phase of the crash is unaffected by scans of the plasma parameters as long as the pedestal pressure remains constant. The duration of the $2^{\text {nd }}$ phase is strongly anti-correlated with the separatrix density and can be suppressed by the application of nitrogen seeding for divertor cooling. A consistent explanation for the two phases of the ELM crash does not seem possible when considering only the pre-ELM pedestal profiles. The SOL plasma provides the necessary free parameter for a consistent explanation, indicating the importance of the SOL in understanding the ELM crash evolution.
\end{abstract}

\section{Introduction}

In magnetically confined fusion research the focus of the experiments is shifting more and more towards ITER and corresponding reactor relevant machine requirements and plasma operation. Examples are the change from a carbon to a tungsten wall in ASDEX Upgrade (AUG) or the installation of the tungsten-beryllium wall (ITER like wall - ILW) in JET, as well as mitigation of edge localised modes (ELMs) and radiative cooling. Operational scenarios involving radiative divertor cooling with impurity seeding or mitigation of ELMs with magnetic perturbations applied by external coils are intensively studied. In this contribution we discuss the pedestal and ELM characteristics with and without these additional actuators.

A special focus is put on the comparison of discharges from AUG with mainly carbon (AUG-C) and with full tungsten (AUG-W) first wall. Here, the main interest lies in the 
energy confinement which in H-mode is dominated by the pedestal and the ELM behaviour. One can distinguish between two categories of consequences a different wall material can have on ELMs and confinement; first, there are direct consequences like the impurity content, e.g. less low-Z and more high-Z impurities with a metal wall. Second, restrictions/modifications of typical plasma operation triggered by the changed boundary conditions. Changes in plasma operation in general should impact the plasma in the same way regardless of the wall material. Therefore, the first category of direct consequences is more relevant to understand the influence of the wall material on ELMs and plasma performance. Typically, it is difficult to clearly separate between both effects because often necessary comparison experiments are missing in the AUG-C database or reference experiments cannot be reproduced easily in AUG-W.

Previous studies at AUG, for which comparison experiments exist - mainly at low triangularities - showed similar confinement factors [1-4]. However, in the broader database of AUG high performance discharges the trend is that in AUG-C the maximal achievable energy confinement was above that in AUG-W [1,3]. One possible explanation is the necessity to operate with significant gas puffing for ELM tailoring and thereby impurity control [5] to prevent tungsten accumulation in the plasma center which can lead to a radiative collapse of the plasma [1. High levels of gas puffing were reported to deteriorate confinement both in devices with a carbon and with a metal wall [6]8]. Due to the increased gas puffing, discharges in carbon with very high performance $H_{98(y, 2)} \sim 1.5$ at very low plasma collisionality were difficult to reproduce [3. These were only recently reproduced and, therefore, are not included in the presented study.

It is an active field of research whether changes in the plasma operation can already explain observed differences in energy confinement between carbon and metal machines or additional phenomena need to be considered. It is long known that high- $Z$ wall materials can cause severe energy losses when accumulating in the plasma center, however, in normal plasma operation the high-Z impurity content is kept low enough. The low-Z impurities carbon and nitrogen were reported to show an impact on global confinement when they were artificially seeded into a plasma with very low natural impurity content [9 11]. The variation of impurity content in these seeding experiments is similar to experiments performed in carbon and metal devices, therefore, it was argued that an effect on confinement could have the same origin in both types of comparison experiments [10. There are studies where the impurity content could indeed provide an explanation for the observed plasma performance, however, other studies with similar differences in the impurity content do not show any change of the energy confinement. Examples for the latter category are low triangularity discharges at constant gas puff levels which showed comparable plasma performance with carbon and metal wall in AUG and JET [2,8]. The former are, in particular, high triangularity JET-ILW baseline discharges [8, 10].

Most of the cited studies were performed using a database approach which is well suited to identify general trends. This paper tries to identify the different actuators by a careful selection of comparison experiments. Instead of global confinement, the focus is on the pedestal structure and the ELM behavior. In section 2 the pedestal structure in carbon is compared to the one in tungsten without gas puffing at low triangularity. The influence of different deuterium fuelling levels is then presented. Finally, one example of nitrogen seeding in AUG-W is given. In section 3 it will be shown that ELMs in AUG can exhibit two distinct phases. The duration, size and magnetic signature of these phases are compared for both wall materials and the different gas fuelling scenarios. Section 4 summarises the paper with 


\section{Pre-ELM pedestal profiles}

This section discusses the pedestal parameters, temperature and density, to document any effect of the different wall materials on the plasma edge. The discussion is first restricted to the pre-ELM pedestal profiles which are assumed to be at the MHD stability limit of ELMs. The influence on the ELMs itself will be presented in section 3. First similar discharges without additional gas puffing are compared and then the influence of different gas fuelling scenarios with deuterium and nitrogen is documented.

\subsection{Comparison of carbon and tungsten}

Due to the restrictions of operational space as described in the introduction, the number of good comparison experiments is quite small. Suitable candidates were already described in 12 and [2] with emphasis on global confinement and are now used for a detailed comparison in the pedestal region. The discharges are all $1 \mathrm{MA}$, low triangularity discharges without external gas puffing and heating powers between 6 and $14 \mathrm{MW}$. The differences between the discharges which could not be avoided are a variation of $q_{95}$ from 4.55 in carbon to 4.85 in tungsten 1 , a reduction in the effective charge number $Z_{\text {eff }}$ from about 2.0 in carbon to 1.5 in tungsten and a different heating mix with NBI and ICRF in the carbon case and NBI and ECRH in the tungsten case. The ICRF is replaced by ECRH and one additional beam source. This results in a larger core rotation, though, the difference in rotation vanishes towards the edge. Despite the different heating mix, the heat flux profiles - determined with the transport code TRANSP [13] - are found to be the same for the ions on the whole radius and for the electrons on the outer half radius.

To illustrate the effect of the different wall materials two phases with moderate $(8 \mathrm{MW})$ and high (13 MW) heating power are compared. Figure 1 shows ELM synchronised preELM profiles composed of data from electron cyclotron emission (ECE) [14, Li-beam [15] and Thomson scattering (TS) [16] measurements. For the ELM synchronisation profile data in a $2 \mathrm{~ms}$ interval before the ELM onset is used, for the presented cases this corresponds to about the last $25 \%$ of the ELM cycle. The profiles illustrate the different behaviour of electron temperature and density. In the tungsten case the edge plasma is colder and more dense. The ion temperatures in the plasma core follow the same trend as the electron temperature, detailed edge measurements of $T_{\mathrm{i}}$ were not available for older discharges with the carbon wall. The electron pressure is the same for $8 \mathrm{MW}$ and also similarly increases with higher heating power. The electron density exhibits several interesting features which are observed for both heating powers in figure 1 (a) and (d). First, the scrape off layer (SOL) density up to the separatrix in carbon is essentially the same as in tungsten. Second, the density gradient in the pedestal is about 25\% larger in tungsten than it is in carbon. Since the pedestal width is almost the same for both wall materials, this results in a roughly $25 \%$ higher pedestal top density. The density gradient length $L_{n \mathrm{e}}=n_{\mathrm{e}} / \nabla n_{\mathrm{e}}$ is reduced for the discharge in tungsten. These are indications that the particle transport in the plasma edge might change. Unfortunately, the particle source profiles cannot be measured and are mainly unknown preventing a definite experimental answer to this question. However, there is no external gas puffing in these discharges so the main particle sources are recycling from

\footnotetext{
${ }^{1}$ Throughout this paper one focus is on the comparison of plasma operation in AUG with carbon and full tungsten wall. To allow for more fluid reading terms like carbon/tungsten case or discharge will be used. This refers only to the wall material, the discharges were run all with deuterium as main gas.
} 

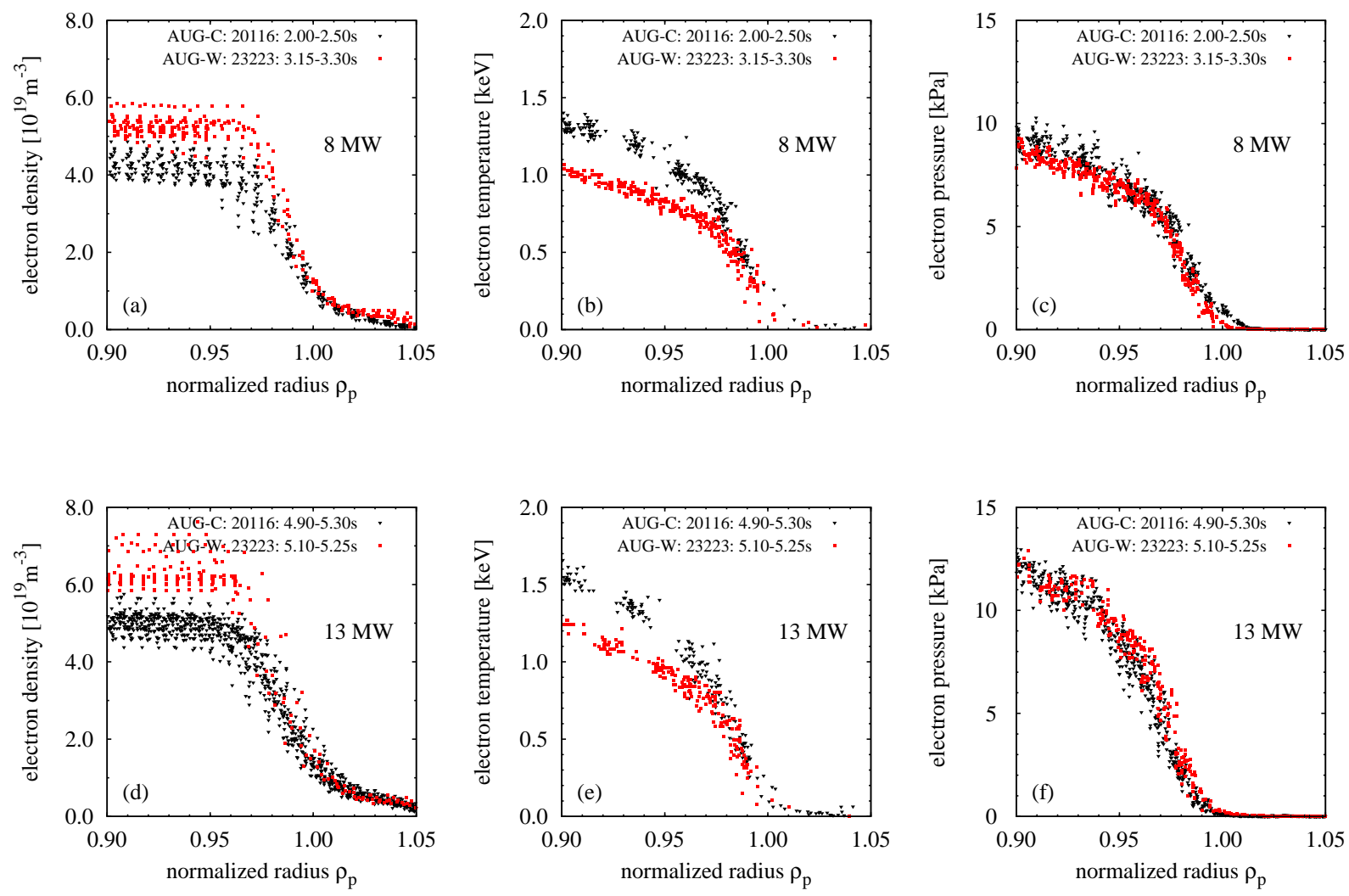

FIG. 1: Edge pedestal profiles of electron temperature, density and pressure compared for similar discharges in carbon $(\mathbf{\nabla})$ and tungsten $(\mathbf{\square})$ and different heating power, $8 \mathrm{MW}$ in the top row and $13 \mathrm{MW}$ in the bottom row. 

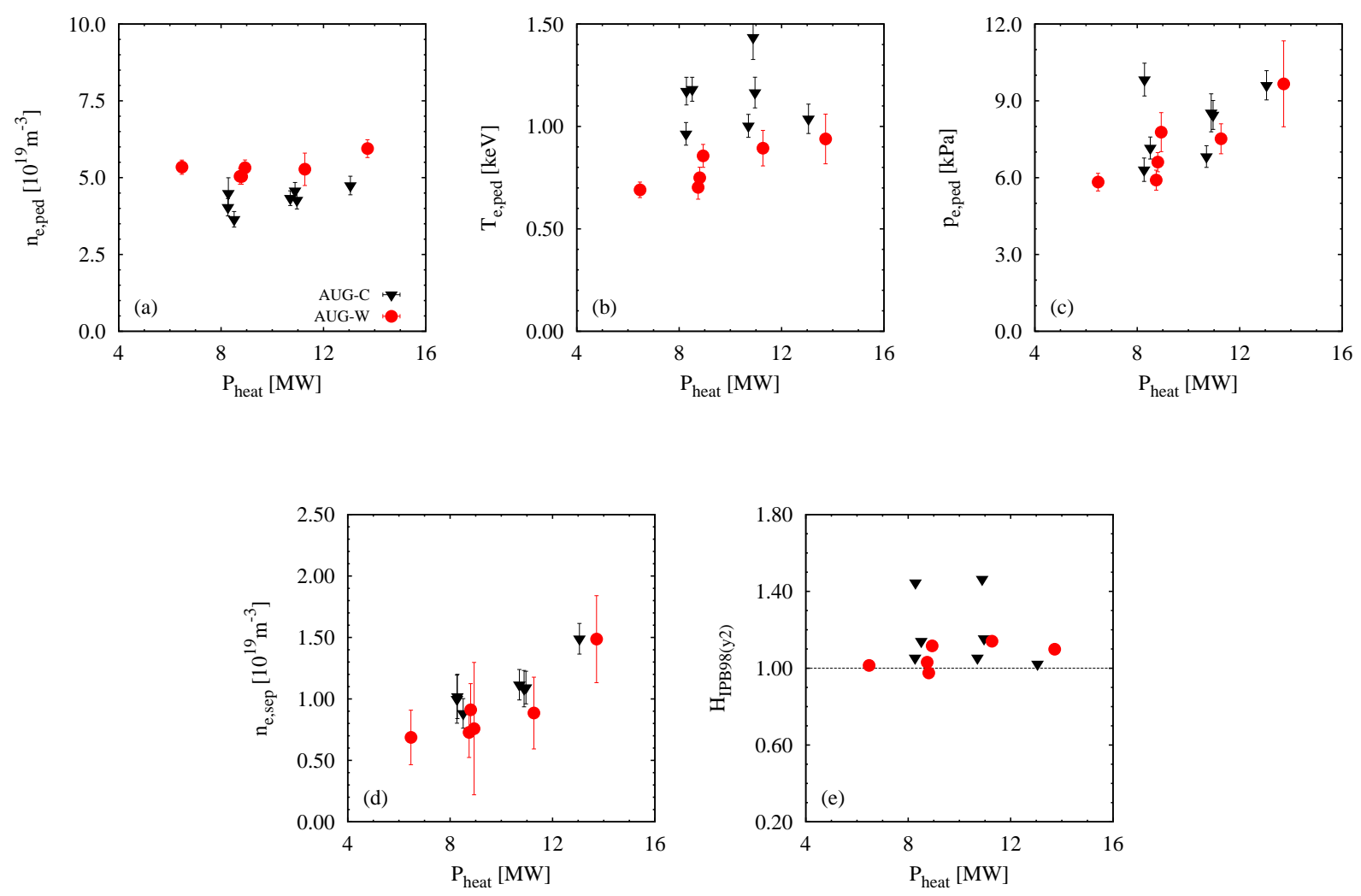

FIG. 2: Pedestal top values of the electron density (a), temperature (b), pressure (c), the separatrix electron density (d) and the IPB confinement factor (e) in carbon ( $)$ and tungsten (-) plotted against the heating power.

the walls and fuelling by the neutral beam injection. Although, the tungsten discharges have one additional neutral beam source to substitute the missing ICRF power, simulations of the beams show fuelling rates of $5 \cdot 10^{20} s^{-1}$ to $6 \cdot 10^{20} s^{-1}$. This difference is $1-2$ orders of magnitude below normal external fuelling levels and therefore too small to account for the density variation. The contribution of the wall recycling to the fuelling might be different which would not be unexpected considering the different wall materials. In principle, carbon is known to have a better pumping capability, i.e. to store more deuterium during the discharge and release it in between discharges [17]. The SOL density, however, is the same in both cases which argues against a strong difference in the wall recycling. Although, it cannot be experimentally verified at this point, an improved particle confinement remains a possible explanation for the higher density in the tungsten case.

Assuming the limiting factor in the pedestal are the ELMs which pose an upper limit to the pressure, as suggested by peeling-ballooning theory [18], the higher density would result in a reduced electron temperature. However, a reversed causality cannot be excluded; if the pedestal temperature is more limited in tungsten and the pressure again clamped by the ELMs the density would be higher.

The plasma response to the increase in heating power from 8 to $13 \mathrm{MW}$ for carbon and tungsten is very similar. If this were not the case, the pedestal stability would be different for carbon compared to the full metal wall. The density increase in the case of higher 
heating power can be attributed to a SOL effect, because the density rises by about $50 \%$ at the separatrix and the neutral pressure measured with pressure gauges in the divertor even doubles. The general picture of the similarity between carbon and tungsten can be drawn with four more discharges of similar type and each with phases of different heating powers. For all these discharges the pre-ELM pedestal profiles are characterised with the two-line method [19] and the results are plotted in figure 2. The density at the pedestal top is larger for all tungsten cases (a), although the separatrix density (d) is similar for both wall materials at constant heating power. The temperature (b) is smaller by the amount needed to have constant $p_{\text {e,ped }}(\mathrm{c})$ at a given heating power. When normalising the confinement time to the ITER98 scaling most of the discharge phases show a similar $H$-factor around 1.1. There are two exceptions with a larger $H$-factor of 1.5 which belong to the same discharge, in [12 the reason for this was attributed to differences in the safety factor profile, namely the shear at mid-radius. The different $q$-profiles influenced the occurrence of instabilities like fishbones and NTMs which can have a significant impact on the core confinement. As indicated in the introduction, in carbon operational windows with very good confinement can be found. Up to now not all of them could be reproduced in the tungsten device. However, in an operational space which overlaps for carbon and tungsten no confinement degradation was found due to the metal wall at low triangularity. In particular, this is true in operation without additional gas puffing. The influence of gas puffing is described in the next section. Observing similar confinement with the same fuelling level in carbon and tungsten is consistent with findings at JET for low triangularity [8]. For high triangularities one has to distinguish between baseline $\left(q_{95}<3.5, \beta_{\mathrm{N}} \sim 1.0-2.0\right)$ and hybrid $\left(q_{95}>3.5, \beta_{\mathrm{N}} \sim 2.0-3.0\right)$ discharges [8] hybrids typically have lower collisionalities than baseline plasmas. While for the hybrids the global confinement is again comparable both in carbon and ILW, the baseline plasmas have strongly reduced confinement in the ILW, even for similar fuelling levels. In both cases the pedestal contribution to the global confinement changes from JET-C to JET-ILW. A consistent explanation for these observations is still missing.

\subsection{Influence of external gas puffing}

The examples presented in the previous section are fairly special cases and, although, they nicely illustrate the similarities between the $\mathrm{C}$ and $\mathrm{W}$ discharges without gas puffing, they are not representative for most of the AUG-W operation. Typically, gas is puffed into the machine to increase the ELM frequency and thereby moderate the impurity content in the plasma [5]. The influence of additional gas puffing on the pedestal profiles is documented in the following section.

\subsubsection{Deuterium}

To avoid a bias by plasma shape or current, discharges with a similar discharge setup as in section 2.1 are selected which have an additional external gas puff. The power scan is achieved only by increasing the NBI power, the ECRH is constant at about $1.2 \mathrm{MW}$ for all cases. The nominal gas puff levels are classified as follows, finite puff rates below $5 \cdot 10^{21}$ electrons/s are labeled "low", between $5-10 \cdot 10^{21}$ electrons/s "medium" and larger gas puffs "high". The influence of these levels on the plasma density can vary with gas puff location - divertor or midplane - and with the machine condition for a particular discharge. Since all discharges used here are from the same experimental campaign, additional factors like a different first wall structure, i.e. changes in pumping/fuelling channels, do not bias the analysis. However, this can be an issue when comparing discharges from different campaigns. Therefore, the values above do not provide a general classification. 

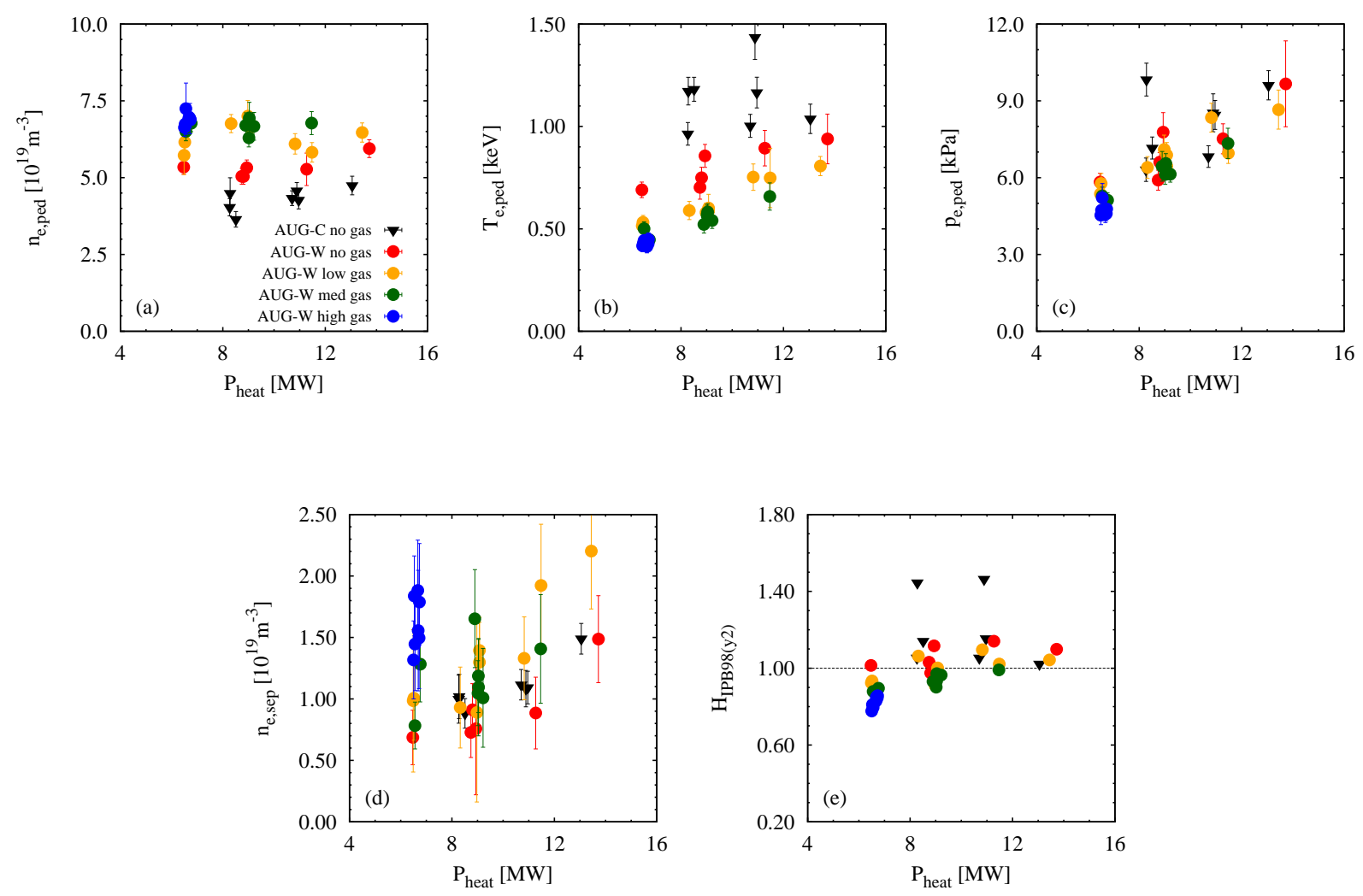

FIG. 3: Pedestal top values of the electron density (a), temperature (b), pressure (c), the separatrix electron density (d) and the IPB confinement factor (e) plotted against the heating power without

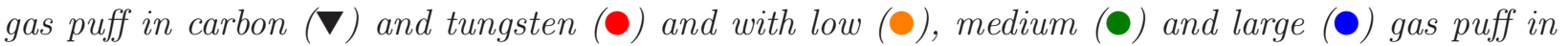
tungsten.

The results of these gas scan experiments are shown in figure 3. By adding additional gas the plasma density increases compared to the unpuffed cases. The electron temperature at the pedestal top decreases while the pedestal electron pressure shows no systematic deviation due to the gas puffing. The separatrix electron density shown in figure 3 (d) is increasing with the external gas puff for most of the cases. This is also consistent with a larger dataset of AUG discharges presented in [20]. Except for the points with the high gas puff the $H$-factor shown in figure 3 (e) is fairly constant regardless of the density. The discharges with high gas puffing all deviate from the scaling towards a $20 \%$ lower $H$-factor. When carbon and tungsten discharges show different confinement factors, this is in most cases accompanied by a much larger gas puff in tungsten which is necessary for stable operation [8,21. Reasons for the lower confinement with very large gas puff might be a changed ELM behaviour as will be discussed in section 3 or the degrading $\mathrm{H}$-mode regime at high densities as observed at AUG [7]. The observation of how external particle sources impact the separatrix density, supports the hypothesis that the higher density in the un-puffed tungsten discharges originates in the pedestal.

The trend between heating power and pedestal pressure shown in figure 3 (c) and its independence of temperature and density allows an estimate for the pedestal pressure, based on engineering variables, to be made. For a larger set of AUG discharges, which includes the same data set as before with additional data at different plasma current and magnetic 


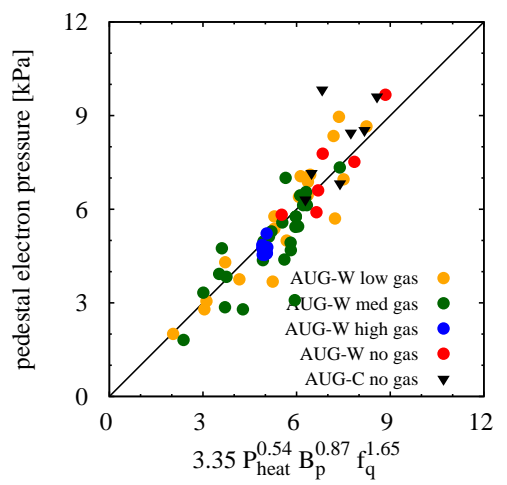

FIG. 4: Measured pedestal top electron pressure plotted against an estimate with engineering variables - without gas puff in carbon $(\mathbf{\nabla})$ and tungsten (-) and with low (-), medium (-) and large (-) gas puff in tungsten.
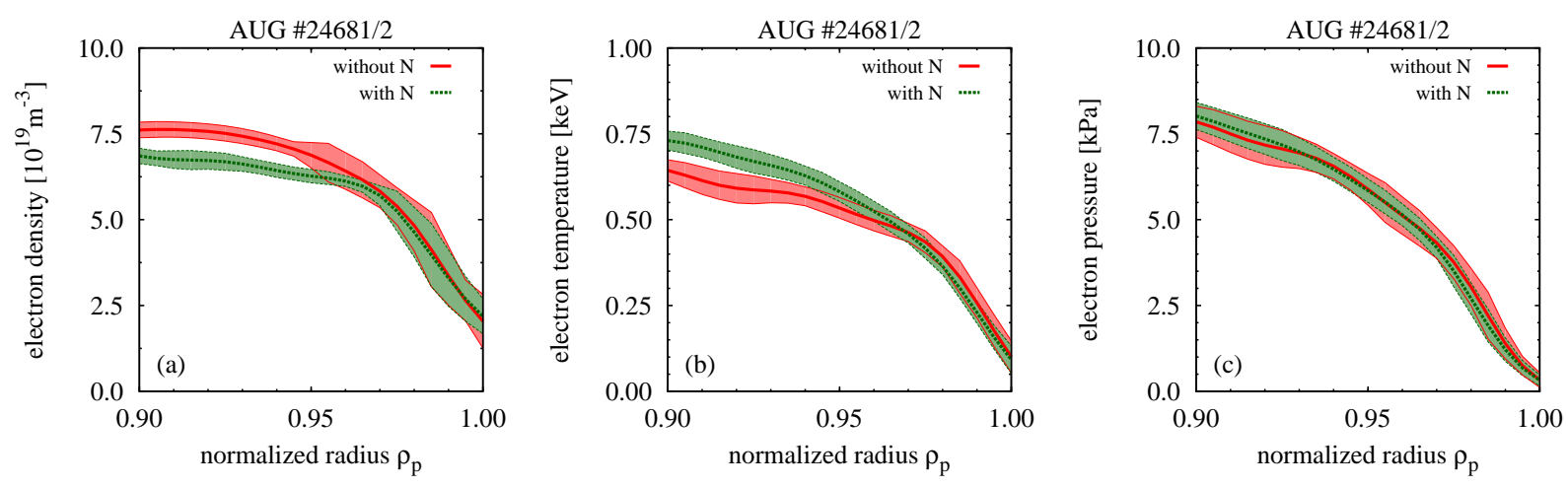

FIG. 5: Fits to electron density (a), temperature (b) and pressure (c) for the case with nitrogen (---) and without (-). [22]

field, a good estimate for the pedestal pressure in AUG H-mode discharges is found with

$$
p_{\text {e,ped }}[\mathrm{kPa}]=3.35 \cdot P_{\text {heat }}^{0.54}\left\langle B_{\mathrm{p}}\right\rangle^{0.87} f_{\mathrm{q}}^{1.65}
$$

where $P_{\text {heat }}[\mathrm{MW}]$ is the total heating power including the ohmic contribution but without correction for the radiation, $\left\langle B_{\mathrm{p}}\right\rangle=\mu_{0} I_{\mathrm{p}} / l[\mathrm{~T}]$ is the flux surface averaged poloidal magnetic field at the separatrix with the separatrix contour length $l, f_{\mathrm{q}}=q_{95} / q_{\text {cyl }}$ as discussed in [20]. The comparison of this estimate with experimental data is shown in figure 4 and illustrates a decent match for pedestal pressures between 2 and $10 \mathrm{kPa}$. The discharge with modified current profile discussed in section 2.1 is the prominent outlier at a pedestal electron pressure of $10 \mathrm{kPa}$, indicating that these effects are not covered by this estimate. In section 3 it will be shown that for an improved estimate the heating power should be corrected by the ELM power losses and likely by the core radiation. This means the governing parameter for the pedestal top might be the power leaving the confined region by steady state transport mechanisms. This is not applied in the estimate in Eq. (1) because this would include physics parameters in the scaling which are in general not known before the discharge. 


\subsubsection{Nitrogen seeding}

The consequences of nitrogen seeding on the pedestal profiles have been thoroughly discussed e.g. in [3, 10,22, 23]. Here, a short overview is given in the context of the observations made in the previous sections. In particular, the focus is put on an example with low heating power, $8 \mathrm{MW}$, and only marginal confinement improvement $(<10 \%)$. This is done because the pedestal pressure is altered only little while the ELMs already change significantly as will be discussed in section 3. In general, the pedestal response to the nitrogen seeding is more diverse, this can be seen in the references above, a common observation in most cases is a reduction of the ratio $n_{\mathrm{e}} / T_{\mathrm{e}}$. It has to be noted that when increasing the amount of seeded nitrogen at a certain point divertor detachment is triggered and the pedestal and ELM characteristics discussed in this contribution are not observed any more [24. The amounts of nitrogen seeded in the discharges presented here are well below that limit.

In the example shown in figure 5 the electron density (a) decreases while the electron temperature (b) increases resulting in a constant electron pressure. It has been argued that the nitrogen might act as substitute for the missing carbon impurities in the tungsten discharges [10]. The trend in the pedestal top values is similar as observed for the pedestal comparison between tungsten and carbon. The $Z_{\text {eff }}$ is also increasing from 2.0 to 2.5 with the nitrogen seeding. However, within the uncertainties no difference in the pedestal gradients is observed for $n_{\mathrm{e}}$ and $T_{\mathrm{e}}$ as was the case in section 2.1. Rather, the density pedestal gets narrower. The additional impact of nitrogen seeding on the divertor plasma and the fuelling efficiency into the main plasma prevents a more definite answer regarding the comparability between carbon and nitrogen as main impurity. Further, there are significant differences in the ELM behaviour which will be discussed in the next sections. A further complication is the necessity of deuterium puffing in nitrogen seeded discharges. Without the deuterium puff too much nitrogen would enter the main plasma and prevent stable operation. Therefore, any comparison experiment with nitrogen seeding includes also a deuterium puff. Depending on the target plasma nitrogen seeding improves the confinement by up to $50 \%$. The exact level of improvement seems to depend on the confinement of the reference discharge, the largest improvement up to now was found in a discharge with high deuterium fuelling and moderate confinement $H_{98, y 2} \sim 0.8$ in the reference [10]. A reference discharge with half the amount of deuterium fuelling and a normal confinement of $H_{98, y 2} \sim 1.0$ shows a confinement improvement of about $20 \%$, resulting in confinement factors for $\mathrm{N}$ seeded discharges between $1.0-1.3[3]$.

\section{$3 \quad$ ELM characteristics}

This section shows that a typical type-I ELM crash in AUG consists of two distinct phases. The characteristics of the two phases is discussed by means of the comparison experiments presented in the previous sections as well as a large set of AUG standard Hmodes. Perturbations of the magnetic field due to the ELM crash will be introduced as the main tool for the characterisation of the two ELM crash phases.

\subsection{Nitrogen seeding}

The existence of two distinct phases in an ELM crash was first reported in [22]. With the application of nitrogen seeding it is possible to suppress the second phase of the ELM crash, while leaving the first phase mainly unchanged. This concept is illustrated with time traces of magnetic pick-up coils, divertor current measurements and edge soft-x ray radiation in figure 6. The two phases of the crash are highlighted by colored shading in green and 


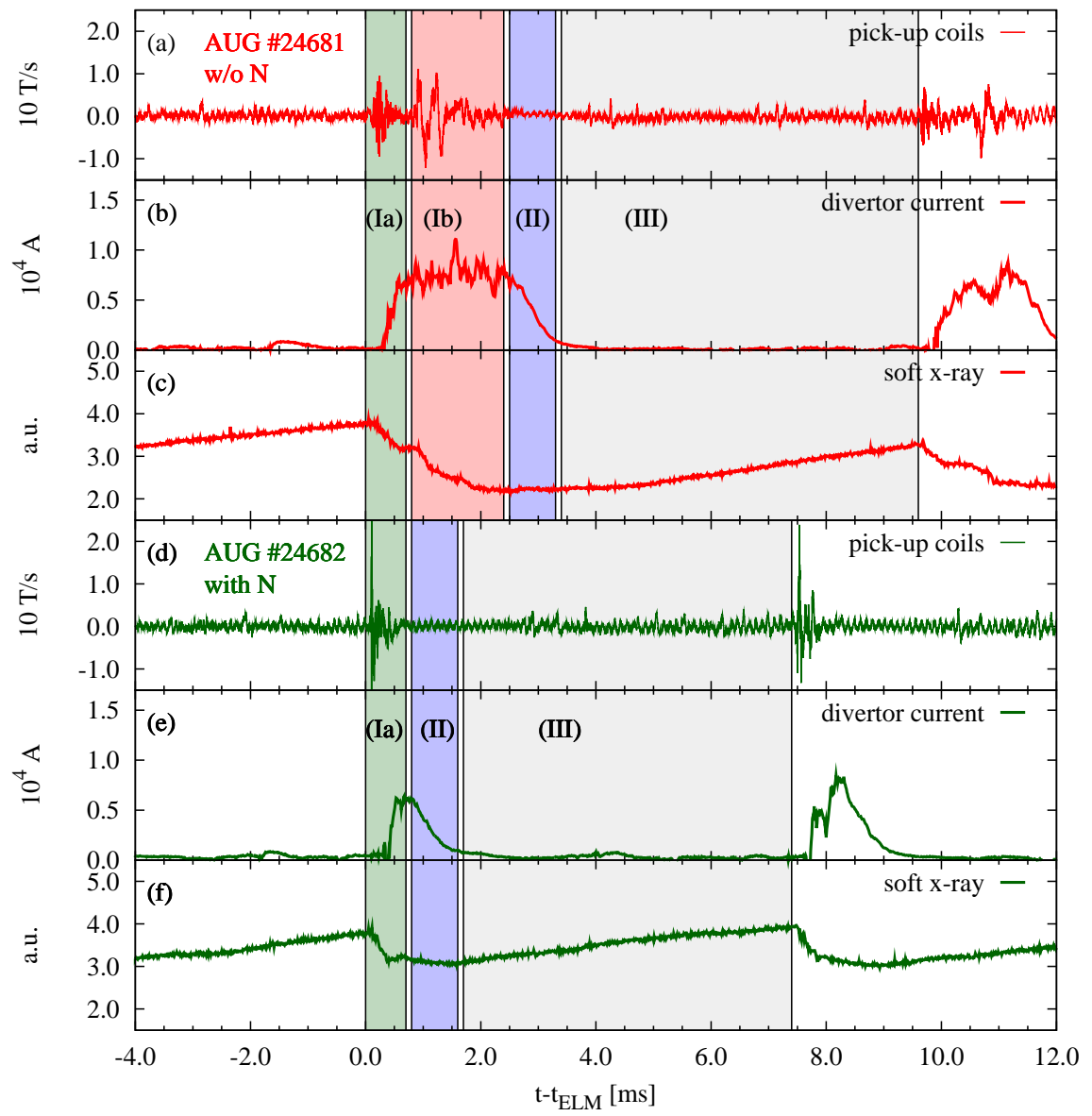

FIG. 6: The time traces of pick-up coils (a), the divertor current (b) and edge soft x-ray emission (c) are shown for the reference case without nitrogen gas puff. (d)-(f) show the same diagnostics in the presence of a nitrogen gas puff. Different phases in the ELM cycle are highlighted - green and red for the ELM crash, blue for the intermediate phase and grey for the recovery phase. [22] 

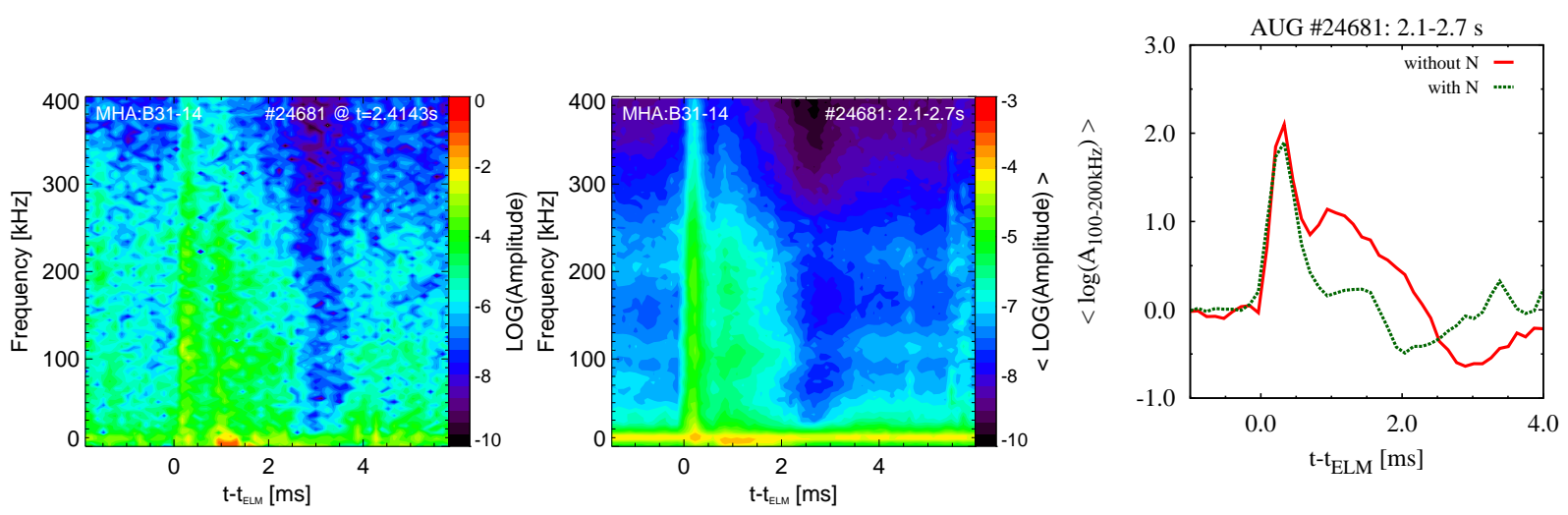

FIG. 7: Spectrogram of perturbations in the magnetic field due to an individual ELM with two phases (a), a characteristic spectrogram averaged for 52 ELMs and the averaged logarithmic amplitude between 100-200 kHz (c) for the case with only the $1^{\text {st }}$ phase (---) and with 1. and $2^{\text {nd }}$ phase of the ELM $\operatorname{crash}(-)$.

red along with the other phases of the ELM cycle. The first ELM phase exhibits a high frequency perturbation in the magnetic field. The second phase has a strong low frequent component which is also overlaid with a faster modulation. These characteristics are best analysed in a spectrogram of the pick-up coil measurements which is shown in figure 7 (a) for an individual ELM. The average over multiple ELMs (b) pronounces the main features more clearly: $1^{\text {st }}$ phase of a few $100 \mu$ s with strong broadband fluctuation between $0-400 \mathrm{kHz}$, the $2^{\text {nd }}$ phase of a duration of up to $2 \mathrm{~ms}$ and broadband fluctuations up to $250 \mathrm{kHz}$ with a strong low frequent component below $10 \mathrm{kHz}$. Because it is difficult to compare multiple spectrograms an averaged amplitude between $100-200 \mathrm{kHz}$ is normalized to the pre-ELM value and used for comparison of different ELM types. Low frequencies have to be excluded because they often show perturbations of the magnetic field which are not ELM related. In figure 7 (c) the averaged amplitude is about one order of magnitude larger for the $1^{\text {st }}$ compared to the $2^{\text {nd }}$ phase. The $1^{\text {st }}$ phase has the same averaged magnetic signature both with and without nitrogen which strongly suggests it has the same origin in both cases. This is supported by the detailed comparisons of profile measurements and analysis of soft x-ray radiation [22]. On the one hand it was shown in section 2.2.2 that the pedestal pressure does not vary significantly with nitrogen seeding in the presented case. On the other hand, the divertor/SOL plasma is altered significantly [25]. This suggests an influence of the divertor/SOL plasma on the $2^{\text {nd }}$ phase while the $1^{\text {st }}$ phase is the same at constant pedestal pressure - consistent with peeling-ballooning theory.

This analysis of the magnetic ELM signature is used in the following sections for different reasons besides the capability to easily distinguish between both crash phases. The magnetic measurements are less affected by the plasma density or impurity content than radiation measurements are and the diagnostic was also available for discharges in carbon which is not the case for measurements of the divertor currents.

\subsection{Deuterium gas puff}

To study the influence of the deuterium gas puff on the ELMs a large number of discharges is necessary which have as little as possible variation in heating power and plasma shape. The AUG "standard H-mode" [26] provides the needed high number of similar discharges. 


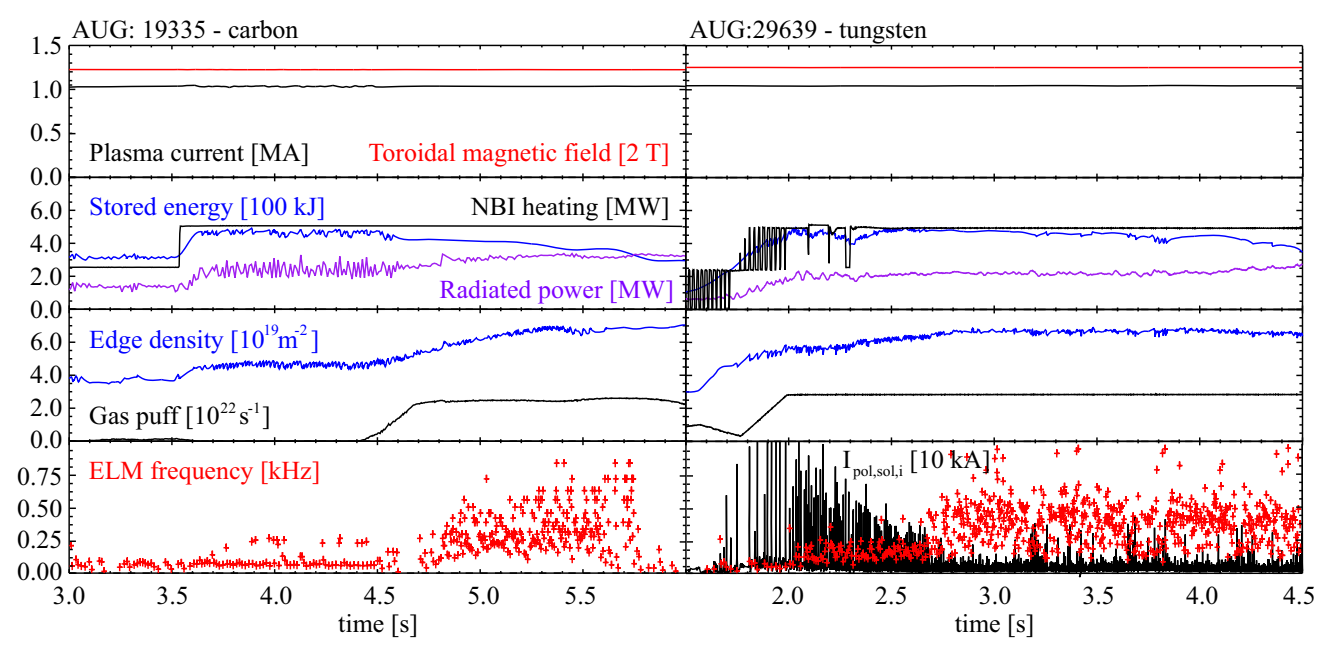

FIG. 8: Time traces of a standard H-mode with carbon wall (left) and a standard H-mode with tungsten wall (right).

It is a standardised type of discharge which was established in AUG to allow long term comparability of plasma operation. It is run at the beginning of nearly every experimental day and for this section discharges from the last 10 years are utilised which span over the upgrade to the full metal wall. In figure 8 time traces of two standard H-mode discharges one in carbon and one in tungsten - are shown which illustrate the typical behaviour. Plasma current is $1 \mathrm{MA}$ and the toroidal magnetic field $-2.5 \mathrm{~T}$. The heating power is ramped up to two neutral beam sources resulting in a stored energy of about $500 \mathrm{~kJ}$ which corresponds to a moderate confinement factor of $H_{98, y 2} \sim 0.9$. The main difference in the parameters of the two discharges is the timing of the deuterium gas puff. In the carbon case there is an early phase with no gas puff, while in the tungsten case the gas puff is present for almost the entire discharge. This is to increase the ELM frequency in order to flush out impurities thus avoid tungsten accumulation. The gas puff level of $2 \cdot 10^{22}$ electrons/s is larger than that of the high gas puff cases discussed in section 2.2.1.

In the ELM frequency the impact of the rising density is nicely illustrated, this is observed similarly in carbon and tungsten. In the beginning ELMs with a frequency of below $100 \mathrm{~Hz}$ are observed, this frequency increases with rising density up to a point where the ELMs occur very irregularly with inter ELM times from 2-10 ms. At the same time the ELMs are becoming smaller which can be observed in the lower right panel of figure 8, where the peak in the divertor currents serves as indicator for the ELM size. The appearance of smaller ELMs at larger electron density is not a new observation and recent discussions can be found e.g. in [27,28] for individual discharges. In this section the high number of analysed standard H-mode discharges $(>200)$, allows to illustrate the influence of the density on ELMs with a broader database and a particular focus on the two ELM phases. The transition to the small or mitigated ELM regime, with losses below $1 \%$ of the total stored energy, will not be discussed further, as these ELMs are too small for the analysis method used here and the reader is refered to [28, 29].

Before the averaging technique can be applied to spectrograms of the magnetic measurements, as described in section [3.1, the ELMs have to be grouped, otherwise the averaging over big and small ELMs would erase most information. For this purpose the peak value of the divertor current during the ELM is utilized. This is only possible for the tungsten standard H-modes where divertor current measurements are available. From the time traces 

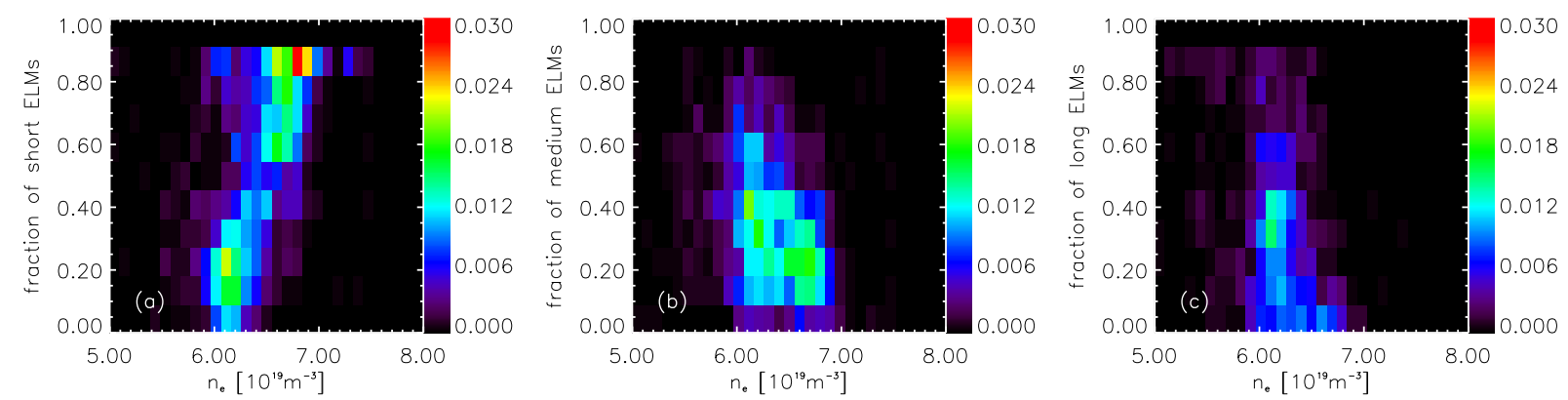

FIG. 9: Histograms of the relative frequency of ELM with different peak divertor currents for line averaged edge densities between $5-8 \cdot 10^{19} \mathrm{~m}^{-3}$ normalized to the number of analysed time intervals.

in figure 8 no indication for a different ELM behaviour due to the wall material can be inferred. Nevertheless, a detailed comparison of carbon and tungsten will be discussed in section 3.3 . For the analysis in the remainder of this section the time between $2.5-3.5 \mathrm{~s}$ is divided into $100 \mathrm{~ms}$ time-intervals which are each analysed individually to track the density evolution.

The ELMs are sorted in four current-intervals between $I_{\mathrm{pol}, \mathrm{sol}}=0,3,5,8, \infty \mathrm{kA}$. The relative frequency of ELMs with these peak divertor currents is illustrated in figure 9 for different densities. The first two current-intervals are combined because they yield similar results. The remaining intervals in $I_{\text {pol,sol }}$ divide the ELMs in short (9a), medium (9b) and long (9r) ELMs. The reason for this labeling is discussed in the next paragraph. Figure 9 shows that in principle ELMs of all sizes can be observed to occur simultaneously in tungsten AUG, however, certain conditions favour one or the other type. The histograms show the relative frequency of each ELM type for different line averaged edge densities [30. Each bin of the histogram gives the percentage of all analysed time-intervals where a given ELM type has a certain relative frequency. Figure 9 (a) shows that below $6.5 \cdot 10^{19} \mathrm{~m}^{-3}$ the majority of time-intervals consist only of about 20\% short ELMs while at higher density $60-90 \%$ of all ELMs are short. Similarly, long ELMs only rarely occur above $6.5 \cdot 10^{19} \mathrm{~m}^{-3}$, while the ELMs with a medium $I_{\text {pol,sol,a }}$ exist for all densities mostly with a relative frequency below $50 \%$. It should be noted that the density of the confined plasma used here is strongly correlated with the separatrix density as shown in section 2.2.1. The only reason for choosing the edge density is that it is more easily measured.

The naming of the four intervals for the peak divertor current in the previous paragraph was already motivated by the analysis of the magnetic spectograms. The averages between $100-200 \mathrm{kHz}$ for ELMs with certain peak divertor currents is shown in figure 10. For the highest peak $I_{\text {pol,sol }}$, therefore the lowest densities, the two phases in the ELM crash are clearly visible, with increasing density and lower $I_{\text {pol,sol }}$ the $2^{\text {nd }}$ phase shrinks until only the $1^{\text {st }}$ phase remains for $I_{\text {pol,sol }}<5 \mathrm{kA}$. Therefore, the ELMs in the two intervals with the smallest $I_{\text {pol,sol }}$ are named short ELMs. When plotting the FWHM of the averaged spectral amplitude one finds that the ELM energy losses determined via the magnetic equilibrium reconstruction scale linearly with the duration of the $2^{\text {nd }}$ phase in the ELM crash. This is shown in figure 11 for standard H-modes between 2009-2011 and 2012-2013 which are both with the full tungsten wall. Although, the ELM energy losses are not the same for the two time periods the increase with ELM duration due to the $2^{\text {nd }}$ phase is comparable as indicated by the linear fits in figure 11. This suggests that the losses which can be attributed to the $2^{\text {nd }}$ phase of the ELM crash are comparable in both cases. Consequently, the $1^{\text {st }}$ phase of 


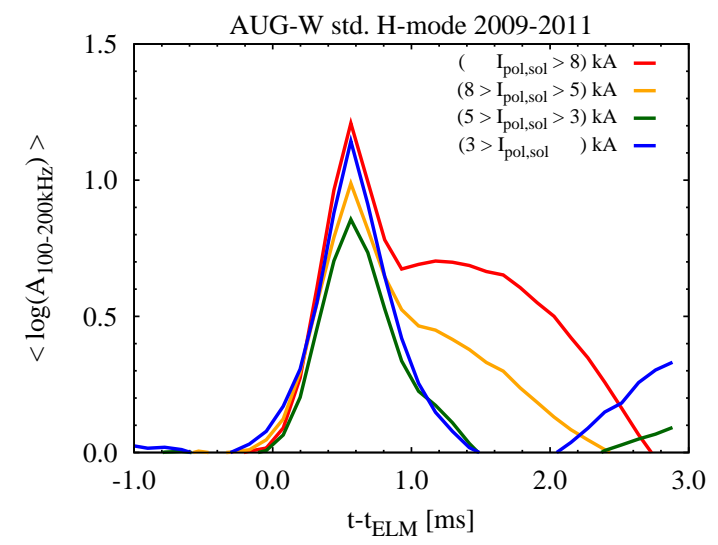

FIG. 10: Averaged logarithmic amplitude of perturbations in the magnetic field between 100-200 $\mathrm{kHz}$, synchronised to the ELM onset and averaged over ELMs of different peak divertor current for AUG standard H-modes between 2009-2011.

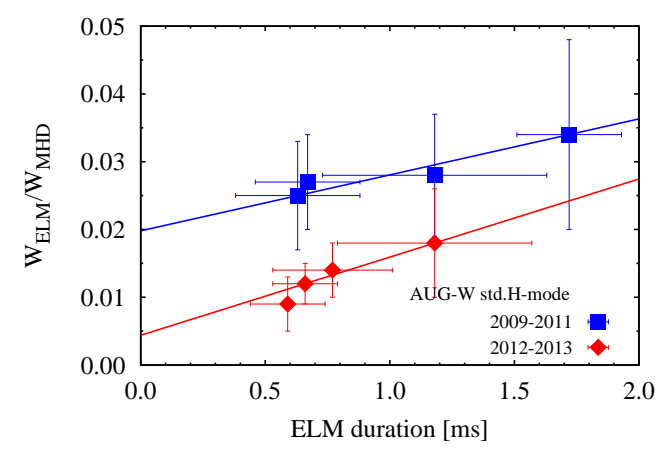

FIG. 11: Relative ELM energy loss plotted versus ELM duration for characteristic ELMs in AUG standard H-modes between 2009-2011 and 2012-2013 $(\bullet)$.

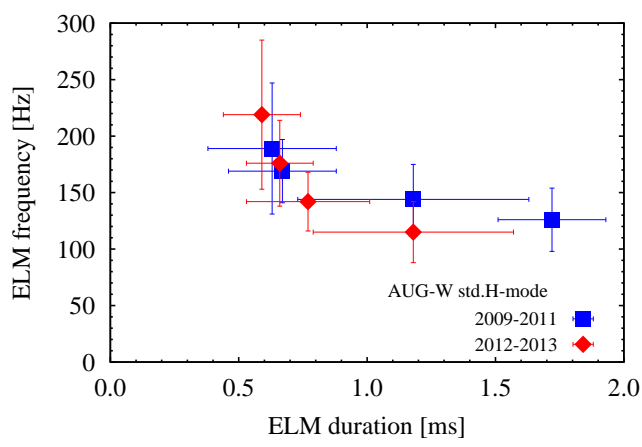

FIG. 12: ELM frequency plotted versus ELM duration for characteristic ELMs in AUG standard H-modes between 2009-2011 (ם) and 2012-2013 $(\bullet)$. 


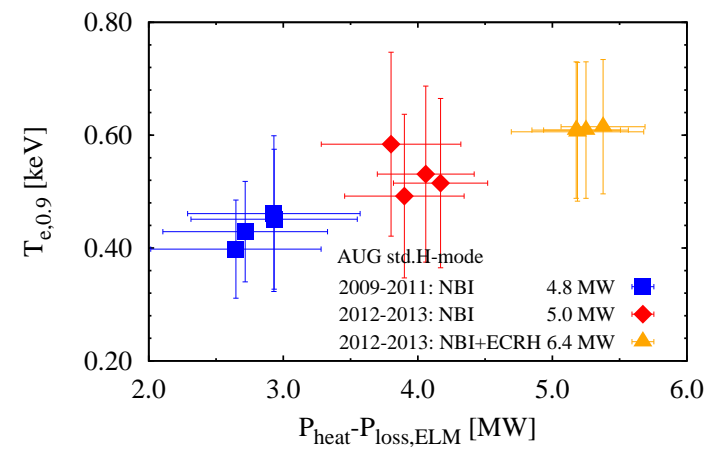

FIG. 13: Edge electron temperature plotted against $P_{\text {heat }}-P_{\text {loss,ELM }}$ for different standard H-modes. For each group of standard H-modes the 4 points correspond to different durations of the $2^{\text {nd }}$ phase as in figure 11.

the ELM is responsible for the difference in the ELM energy losses between the two time periods. The reason for the variation in ELM losses could not be identified up to now. Different ELM frequencies cannot explain the observations because both sets of discharges show similar ELM frequencies as indicated in figure 12, The main differences between the two time periods are a higher $T_{\text {e,ped }}$ at constant $n_{\text {e,ped }}$ for the 2012-2013 discharges and a change in gas puff location, from the midplane to the divertor. The divertor gas puffing is correlated in time with an increase of the midplane neutral pressure. Further, the structure of the first wall was successively altered with the installation of magnetic perturbation coils at the low field side. Indications that the wall clearance of the plasma can influence the ELM behaviour were reported before [31]. To explain the variation in ELM losses dedicated experiments will be performed in the future.

With the available data it can be excluded that a diagnostic artifact in the magnetic measurements, which determines the stored energy, is responsible for the change in ELM losses. This is supported by the observation that the electron temperature follows nicely the trend expected from Eq. (11) when correcting $P_{\text {heat }}$ for the larger ELM power losses $P_{\text {loss,ELM. }}$. Additional evidence is shown in figure 13. There the electron temperature for $\rho_{\mathrm{p}} \sim 0.9$ is plotted against $P_{\text {heat }}-P_{\text {loss,ELM }}$ which is a proxy for the power crossing the separatrix by diffusion or convection, if the core radiation is assumed to be constant. Two results can be inferred from this plot; first the duration of the $2^{\text {nd }}$ phase does not contribute to the ELM power loss. This is seen by the clusters of 4 points each, their proximity illustrates the similarity of the ELM power losses despite the different duration of the $2^{\text {nd }}$ phase. With a longer duration of the $2^{\text {nd }}$ phase and higher energy losses per ELM the frequency of the ELMs reduces keeping the power losses constant. Second, a variation in the ELM power loss is equivalent to a change of heating power of the same magnitude regarding their impact on $T_{\mathrm{e}}$. The ELM power loss can change with the $1^{\text {st }}$ phase of the ELM crash because the ELM frequency is independent of the energy loss in this phase. The difference in energy loss at constant frequency sums up to a power loss which is larger by $\sim 1$ MW for the 20092011 standard H-modes. This is correlated with a reduction of the temperature by $0.1 \mathrm{keV}$. Additional ECRH heating of $\sim 1 \mathrm{MW}$ in some of the 2012-2013 standard H-modes results in a comparable temperature increase of $\sim 0.1 \mathrm{keV}$. 


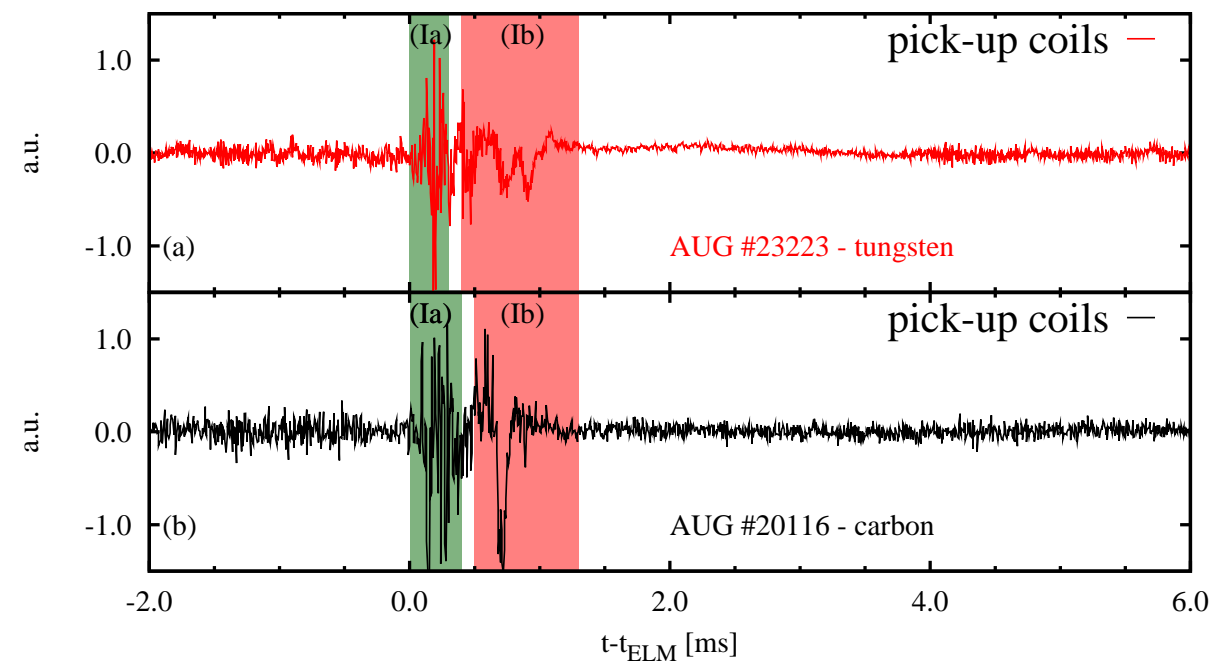

FIG. 14: Time traces of the pick-up coil signal for individual ELMs for the comparison discharges between carbon and tungsten wall discussed in section 2.1.

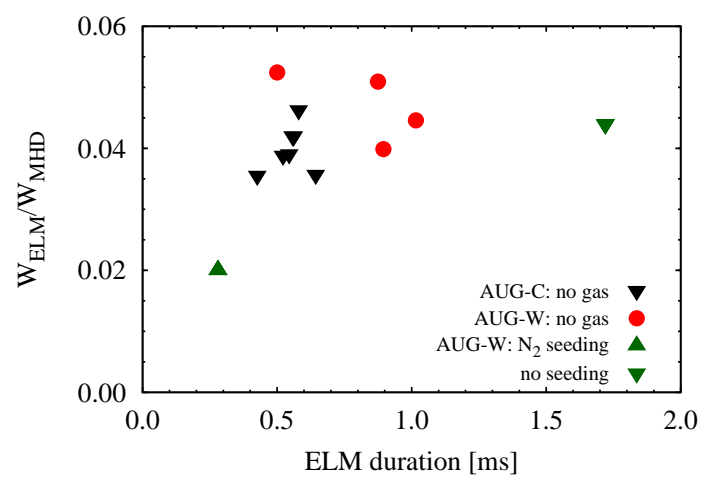

FIG. 15: Relative ELM energy loss plotted versus ELM duration for unpuffed discharges in carbon $(\boldsymbol{\nabla})$ and tungsten $(\mathbf{\bullet})$. The changes due to nitrogen seeding are added for comparison $(\mathbf{\Lambda}, \boldsymbol{\nabla})$. 


\subsection{Carbon and tungsten}

In the previous sections it was shown that the ELM consists of two phases in tungsten and that the duration of the $2^{\text {nd }}$ phase correlates either with $n_{\mathrm{e} \text {,ped }}$ or with $n_{\mathrm{e}, \mathrm{sep}}$. This section will show observations of the two crash phases in carbon and bring arguments for the separatrix density being the dominant quantity for the duration of the $2^{\text {nd }}$ phase of the ELM crash.

The ELM characteristics of the carbon-tungsten comparison discharges discussed in section 2.1 is also useful for the ELM comparison. In this comparison $p_{\text {e,ped }}$ was constant and $n_{\mathrm{e}, \text { ped }}$ increased while $n_{\mathrm{e}, \mathrm{sep}}$ remained constant. In figure 14 it is shown that the ELMs do not change under these conditions despite the different wall materials. In carbon the trace of the magnetic pick-up coils shows the two distinct ELM phases, with the same length as in the tungsten reference. The ELM duration is shown in figure 15 supporting the observation that neither the ELM energy losses nor the duration of the two phases exhibit significant differences due to the wall material. As comparison also the nitrogen seeded discharge with its reference is plotted in green. In the nitrogen seeding experiment $p_{\text {e,ped }}$ was also the same for both cases, but the ELM characteristics changed significantly. $Z_{\mathrm{eff}}$ and $n_{\mathrm{e}} / T_{\mathrm{e}}$ changed similarly in both comparisons which suggests that these quantities do not play a dominant role in the suppression of the $2^{\text {nd }}$ phase in the ELM crash. The main difference between the carbon/tungsten and $\mathrm{N}$ seeding/no seeding is the divertor and SOL plasma. The former showed similar SOL densities, while in the latter the nitrogen seeding is known to have a strong impact on the divertor and SOL plasma.

One can distinguish between a hot, thin and a thick, cold inter ELM SOL/divertor plasma where the latter is achieved with strong deuterium puffing or nitrogen seeding [32]. Nonlinear 2D reduced magnetohydrodynamic simulations suggest a stochastisation of the plasma edge due to ELMs [33, 34] and thereby mixing the pedestal and SOL plasma. Even when starting with the same pedestal pressure, the pedestal-SOL mixture can be quite different and influence the evolution of the ELM crash. This could explain why the ELMs are not affected in the carbon/tungsten comparison with the same pedestal pressure profiles and similar SOL plasmas. At the same time nitrogen seeding changes the ELMs significantly, despite unchanged electron pedestal pressure profiles, because with the seeding the SOL plasma becomes thicker and cools down. Detailed nonlinear MHD simulations are in preparation to test this hypothesis.

\section{Conclusions}

This paper gave an overview of pedestal profiles of temperature and density and the ELM crash for AUG H-mode operation at low triangularity with different boundary conditions, namely different wall materials: dominantly carbon and full tungsten; and different external gas fuelling: without external gas puffing and with gas puffing of deuterium and nitrogen.

It was shown that for all these different boundary conditions the pedestal electron pressure can stay essentially constant when the heating power, plasma current and plasma shape were not changed. In particular, operation with the full metal and the carbon wall showed no significant differences in plasma performance without gas puffing at low triangularity. The effect of the boundary conditions manifests in a different composition of the pedestal pressure, namely the electron temperature and density. In tungsten the electron density is significantly larger than in a comparison discharge in carbon - both without gas puffing. The higher density was caused by a steeper density gradient in the pedestal. The steeper gradient could be due to improved particle confinement in the pedestal for the tungsten 
case. Although accurate measurements of the particle source profiles are missing to support this hypothesis experimentally, it remains a possible explanation. This is supported by the observation that external gas sources influence the separatrix density which was not observed in the carbon-tungsten comparison where the density at the separatrix was fairly similar. The higher natural density is one reason why certain high performance regimes observed in the carbon device at low collisionality were only recently reproduced with the tungsten first wall.

The nitrogen seeded case was specially chosen to exhibit constant electron pressure and still show a significant impact on the ELM characteristics. In general, the pressure is not constant, in particular, for higher heating power a larger pedestal pressure is observed with the seeding.

The impact of the impurity content on the pedestal stability might be mitigated/enhanced by additional parameters. One candidate could be the collisionality which changes a lot over the presented discharges and is also the parameter changing most in the JET baseline/hybrid comparison at high triangularity [8]. In the pedestal and the core the hybrid plasmas at low collisionality showed a quite different response to the change from JET-C to JET-ILW compared to the baseline plasmas at higher collisionality.

ELMs in standard AUG discharges exhibit two distinct phases in an ELM crash with different properties. The $1^{\text {st }}$ phase remains the same when the pedestal pressure was not changed. The $2^{\text {nd }}$ phase can be reduced in size by deuterium fuelling and increasing of the separatrix density or it can be suppressed completely by applying nitrogen seeding. Both phases are observed also in carbon discharges with similar size as in the tungsten comparison discharge. There were no indications found that ELMs in carbon and tungsten are fundamentally different. Also their response to additional deuterium puffing and the resulting density increase is similarly observed in standard H-modes in carbon and tungsten.

These observations of the pedestal structure and ELM behavior do not suggest that nitrogen simply acts as substitute for carbon as impurity in the pedestal. Nitrogen seeding suppresses the $2^{\text {nd }}$ ELM crash phase while it is unaffected by carbon. In the AUG-C/AUG$\mathrm{W}$ comparison carbon reduces the density gradients while nitrogen seeding in $\mathrm{AUG}-\mathrm{W}$ is accompanied with smaller pedestal widths at constant gradients. However, the nitrogen seeding also has a strong impact on the SOL plasma, which is not similarly pronounced in the AUG-C/AUG-W comparison.

Both phases of the ELM crash appear to be largely independent of each other. The $2^{\text {nd }}$ phase can have different size and duration while the $1^{\text {st }}$ phase remains unaffected and vice versa. Differences are found in their correlation with ELM power losses. Changes in the duration and size of the $2^{\text {nd }}$ phase anti-correlate with the ELM frequency resulting in the same ELM power loss. In standard H-mode plasmas of two consecutive campaigns different sizes were observed for the $1^{\text {st }}$ phase at constant frequency and thereby reducing the ELM power losses. The difference in ELM power loss was found to have a comparable impact on $T_{\mathrm{e}}$ as the same amount of power would have by means of external heating.

The reason causing the reduction in power loss, could not be identified with the available data. Influences on the ELM size like pedestal collisionality or plasma shape can be excluded as reason. The plasma shape did not change in the standard H-modes and the collisionality was varied significantly in different comparison experiments without an impact on the $1^{\text {st }}$ phase of the ELM crash.

When only considering the pre-ELM pedestal parameters, a general explanation for the ELM characteristics does not seem possible. Adding the SOL plasma into the description 
allows to provide a hypothesis: The $1^{\text {st }}$ phase in the ELM crash is set by a stability limit of the pedestal. With the $1^{\text {st }}$ phase the plasma edge becomes stochasticised, thereby mixing the pedestal and SOL plasma - which is supported by reduced magnetohydrodynamic simulations [33, 34]. The $2^{\text {nd }}$ phase is then not governed by the pre-ELM pedestal plasma alone, but the mixture with the SOL.

The observed coupling between the edge temperature and the ELM power loss corrected heating power could influence the quality of extrapolations for the pedestal temperature which are only based on the nominal heating power in future reactor like machines. However, the general trend of these extrapolations should still be correct when the ELM power losses do not vary too much.

The observations about different ELM phases could also impact predictions and extrapolations to future machines. Considering the need for radiative cooling in the SOL and divertor with nitrogen seeding [25] one would expect - based on above hypothesis - the $2^{\text {nd }}$ ELM crash phase to be suppressed by the cold, dense SOL plasma. At the same time no effect on the $1^{\text {st }}$ phase was observed. This would mean extrapolations of ELM sizes might be misleading if they are based on data which does not properly separate both phases of the ELM crash.

Acknowledgments The authors want to thank M. Beurskens, F. Ryter and J. Schweinzer for fruitful discussions about nitrogen seeding and standard H-modes. This work has been carried out within the framework of the EUROfusion Consortium and has received funding from the European Union's Horizon 2020 research and innovation programme under grant agreement number 633053. The views and opinions expressed herein do not necessarily reflect those of the European Commission. References

[1] GRUBER, O. et al., Nuclear Fusion 49 (2009) 115014.

[2] MAGGI, C. et al., Nuclear Fusion 50 (2010) 025023.

[3] SCHWEINZER, J. et al., Nuclear Fusion 51 (2011) 113003.

[4] NEU, R. et al., Journal of Nuclear Materials 438 (2013) S34.

[5] PüTTERICH, T. et al., Journal of Nuclear Materials 415 (2011) S334.

[6] SAIBEnE, G. et al., Plasma Physics and Controlled Fusion 44 (2002) 1769.

[7] BERNERT, M., The H-mode density limit at the ASDEX Upgrade tokamak, submitted to PPCF.

[8] BEURSKENS, M. N. A. et al., Nuclear Fusion 54 (2014) 43001.

[9] RATHGEBER, S. K. et al., Plasma Physics and Controlled Fusion 52 (2010) 095008.

[10] BEURSKEnS, M. N. A. et al., Plasma Physics and Controlled Fusion 55 (2013) 124043.

[11] GIROUD, C. et al., Nuclear Fusion 53 (2013) 113025.

[12] STOBER, J. et al., Nuclear Fusion 47 (2007) 728.

[13] PANKIN, A. et al., Computer Physics Communications 159 (2004) 157.

[14] SALMON, A. N., International Journal of Infrared and Millimeter Waves 15 (1994) 53.

[15] FISCHER, R. et al., Plasma Physics and Controlled Fusion 50 (2008) 085009.

[16] KURZAN, B. et al., Review of Scientific Instruments 82 (2011) 103501.

[17] ROHDE, V. et al., Nuclear Fusion 49 (2009) 85031.

[18] CONNOR, J. W. et al., Physics of Plasmas 5 (1998) 2687.

[19] SCHNEIDER, P. A. et al., Plasma Physics and Controlled Fusion 54 (2012) 105009.

[20] SCHNEIDER, P. A. et al., Nuclear Fusion 53 (2013) 73039.

[21] SCHWEINZER, J. et al., 40th EPS Conference on Plasma Physics, Espoo, Finland (2013) P4.113.

[22] SCHNEIDER, P. A. et al., Plasma Physics and Controlled Fusion 56 (2014) 25011.

[23] DUNNE, M. G., Impact of Te and ne on edge current density profiles in ELM mitigated regimes on ASDEX Upgrade, submitted to NF.

[24] REIMOLD, F., Divertor studies in nitrogen induced, completely detached H-modes in full-W 
ASDEX Upgrade, to be submitted.

[25] KAllenBACH, A. et al., Plasma Physics and Controlled Fusion 52 (2010) 55002.

[26] RYTER, F. et al., Plasma Physics and Controlled Fusion 44 (2002) A407.

[27] BOOM, J. E. et al., Nuclear Fusion 52 (2012) 114004.

[28] RATHGEBER, S. K. et al., Nuclear Fusion 54 (2014) 93011.

[29] BARRERA, L. et al., 40th EPS Conference on Plasma Physics, Espoo, Finland (2013) P4.113.

[30] MLYNEK, A. et al., Review of Scientific Instruments 81 (2010) 033507.

[31] BURCKHART, A. et al., Plasma Physics and Controlled Fusion 52 (2010) 105010.

[32] MüLLER, H. et al., Nuclear Fusion 51 (2011) 073023.

[33] HOLZL, M. et al., Physics of Plasmas 19 (2012) 82505.

[34] KREBS, I. et al., Physics of Plasmas 20 (2013) 82506. 